inangurated. Similar structures may be formed generation after generation, or new structures and new actions may become manifest for the first time. Divergent forms may soon cease to live or may be repeated again and again without change during ages, or the divergence may increase until forms very different in character may be evolved. Prophetic power still hesitates to define with confidence the probable characters which divergent forms to be produced in future years or centuries may assume. The form-evolving power of bioplasm depends upon its vitality rather than upon the substances it takes up as pabulum or the conditions under which it lives, though no doubt in some degree these influence the result

It is not possible to ascertain the dimensions of the most minute living particle which is capable of manifesting all the phenomena which establish its living property or power, but certainly it is considerably less than the one hundred thousandth of an inch in diameter. It is to the central part of such minute living particle that the streams of fluid holding certain substances in solution tend. It is in the central part that certain constituents or elements of the substances newly introduced pass from the non-living to the living state. This is where those mysterious changes occur which result in the communication of vital power from matters already living,-_changes which bave been attributed to the agency of "cell wall," "cell contents," "nucleus," "nucleolus." Some thought the mystery was solved when the word "Protoplasm" was coined; Protoplasm which by some authorities was affirmed to be structureless, by other to exhibit structure; Protoplasm which might be dry or moist, hard or soft, cold or hot, roasted or boiled, dead or living-and yet retain its protoplasmic properties, assimilating, converting, constructing, forming, boilding, designing-receiving its properties from solar energy or from the material elements of which it was composed, and possessing molecular machinery which under the influence of its environment performs vital acts and exhibits vital irritability !

The unseen centres of minute particles of bioplasm are the seat of those wonderful vital phenomena which consist of the re-arrangement of the atoms of matter, one result of which is preparation for future chemical and other changes. In the absence of vital action the subsequent formation of structure and the production and arrangement of matters having those definite and peculiar characters and properties by which the various living forms are known to us would have been impossible. It is not probable that if our means of amplification could be increased very many times we should be enabled to see the actual changes which take place in the invisible centres of living particles under the influence of vitality. The matter which is the seat of atomic movements is perfectly structureless and its elements must undergo extensive re-arrangement, portions moving in all directions and mixing with and passing through other portions-all which phenomena in all cases cease at death. In these structureless centres which no eye has yet seen the living matter imparts to new material particles, but without loss, the marvellous vital powers which it received from pre-existing living matter. It is here, too, that changes in vital powers resulting eventually in the development of characters diverging from those manifested by the immediate predecessors, occur. Here, then, is a true evolution-evolution from centres too central to be visible, where structureless, perfectly transparent particles move amongst one another in all directions, their state being diffiuent, from the large proportion of water invariably associated with all matter that lives, and in the absence of which vitality is unknown. To these centres by inherent movements of the living particles streams of fluid, holding in solution certain substances, flow, to some of which vital power is imparted, and which then pass from the non-living to the temporary living state.

But well within the range of actual observation with the aid of magnifying powers of 500 diameters are the actual anatomical elementary units, hundreds and thousands of which are arranged together to constitute but a small part of, for example, an organ of sense. ${ }^{3}$ So delicate and minute are these anatomical units and so closely are they packed that it is not possible to clearly see a single individual anatomical element with its connexions, or to make out with accuracy its several component structural parts. Nor is it possible to separate one from its neighbours and isolate it for accurate examination under high magnifying poxers. We

By the anatomical elementary part or unit is meant the complete arrangement necessary for seeing, hearing, \&c., the actual organ being composed of a multitude of similar elementary parts. do not yet perfectly understand the exact structure or the arrangement of the several parts concerned in feeling, tasting, hearing, seeing or smelling or those taking part in evolving heat, light or electricity. It is certain, however, that in intimate connexion with each anatomical elementary unit are numerous bioplasts which are intimately concerned with the action of the sense organ, and wbich are an indis pensable and indeed a necessary part of the arrangement. These bioplasts are present in great number in all living beings and take part in all vital actions of every organ in ourselves.

Think for a moment of the light-producing elementary parts of the illuminating organ of the glow-worm or the firefly as contrasted with any light giving apparatus constructed by man-or of the anatomical elements taking part in the wonderful movements of the wings or legs of the smallest insects contrasted with any of our motor machines for moving things on earth or water or through air. Can any analogy whatever be pointed out between the structure that has grown and any of the mechanisms made by us-as regards origin, construction, composition or action?

(To be continued.)

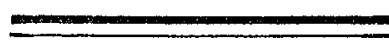

THE

ELIMINATION OF BACTERIAL TOXINS BY MEANS OF THE SKIN, WITH ESPECIAL REFERENCE TO THE PRESENCE OF TUBERCULIN IN THE SWEAT

\section{OF PHTHISICAL} PATIENTS.

BY ALFRED SALTER, M.D., B.S., D.P.H. LOND., GOLI RESEARCH SCHOLAR IN PATHOLOGY, AND ASSISTANT BACTERIO LOGIST, AN'ITOXIN DEPARTMENT, BRITISH INSTITUTE OF PREVENTIVE MEDICINE.

THE body, in the words of Bouchard, in its normal as well as its pathological state is both a receptacle and a labora. tory of poisons. These are disposed of by the economy in a variety of ways and by a variety of channels. Amongst the functions performed by the skin that of the elimination of poisonous substances has long been surmised, and in the following paper I hope to demonstrate that the general integument is an important emunctory for many of the toxins manu. factured in the human laboratory by pathogenic organisms. Given appropriate means, it is in the sweat, the main vehicle of skin excretion, that we should expect to be able to find these poisons if they exist. Hitherto, however, the obtaining of this excretion under ordinary conditions in quantity sufficient for experimental purposes has placed almost insuperable difficulties in the way of its examination.

Methods of collecting sweat. -Arloing, ${ }^{1}$ one of the first to investigate by the experimental method the physiological properties of sweat, obtained it by clothing a man in an absorbent woollen garment and setting him to hard and prolonged physical exertion in a hot atmosphere. He then steeped and wrung the vestment in a determined quantity of distilled water and concentrated on a water bath or in vacuo the diluted liquid so obtained. It is obvions that there are endless fallacies involved in such a procedure, and it is only necessary to mention the fact that Arloing himself points out that the sweat of individuals under such circum stances is much richer in toxic principles than that collected under normal conditions. Capitan and Gley's method ${ }^{2}$ consists in enclosing the individual in a hot air chamber and removing the secretion with a sponge. Mavrojannis envelops his subject in an air-tight indiarubber jacket next the skin, furnished with a small outlet tube and stopcock at its lower part. The sweat is then obtained as in Arloing's method by subjecting the individual to hard manual labour and drawing off through the exit tube from time to time the fluid which has gravitated to the lower part of the apparatus.

1 Comptes Rendus de la Société de Biologie, tome jii., No. 35 Dec. 26th, 1896, p. 1107; and ibid., torne iv., No. 19, June 4th, 1897, p. 533. 
It is equally obvious that this method is not applicable to a patient in bed who is sweating moderately or even freely.

For the purpose of collecting sweat under these latter conditions I have had made a double-bulbed tube drawn out at one end to a very fine long capillary point, each bulb having a capacity of about 3 c.c., and a circular tin lid about four inches in diameter fitted around its edges with a small indiarubber air cushion. For a person sweating to the extent of having visible beads of perspiration on his skin or even mere visible moisture it is suficient to streak the fine capillary point of the glass tube over the sweating surface, applying suction with the mouth from time to time to the expanded end in order to draw up the fluid into the first bulb. The interposition of the second bulb prevents any of the liquid ascending the straight part of the tabe. It is surprising what an extremely minute quantity of moisture can be made to enter the capillary limb in this way and frequently in half an honr the bulb can be completely filled. With a patient sweating freely I have several times remored as much as half an ounce of sweat in this time. The tin cap can be placed on the front of the chest where the indiarabber air-cushion will fill up inequalities between the ribs, and two crossed pieces of strapping will keep the whole in position. No evaporation from the skin surface beneath then takes place, and the sweat of ten rapidly collects in beads sufficient to fill several bulbs within a short space of time. There is a further advantage in the apparatus in that the sweat is prevented from being absorbed by contact with the clothes or rabbed off by the movements of the patient. Several of these protecting caps can be placed on the skin at different parts of the abdomen, \&c. They should be warmed to blood-heat before being fixed in position as this will prevent aqueous vapour volatilised from the skin from becoming condensed on their interior.

The night sweats of phthisis. - With this apparatus I have examined experimentally the qualities of the sweat from a number of phthisical patients. The modus operandi consisted in injecting a variable quantity of the sweat in to guinea-pigs artificially rendered tuberculous by the prior inoculation of a living virulent culture of the tubercle bacillus. The animals' weights were taken daily and their temperatures thrice daily from the date of inoculation. In order to register the maximum effect of the sweat toxicity intravenous injection of the liquid into tuberculous rabbits was also practised. I have proceeded in this way with fourteen phthisical patients. All had advanced lung trouble with numerous tubercle bacilli in the sputum. No less than twelve specimen; of sweat obtained by the above method from as many different cases gave a typical tuberculin reaction after inoculation into the diseased animals. A temperatnre chart which I have before me shows the characteristic rise following the introduction of 5 c.c. of sweat. The temperature of the animal, a guinea-pig, was taken every four hours for five days and was always about normal-i.e., about $102^{\circ} \mathrm{F}$. At $10 \mathrm{~A} . \mathrm{M}$. on the sixth day $5 \mathrm{c.c}$. of sweat were injected. By noon the temperature had begun to rise; by 2 P.Mr. it was over $104^{\circ}$ and by 3 P.M. it had reached its maximum-105. $5^{\circ}$. By 4 P.Mr. it had fallen one degree; by 5 PM. it was $103^{\circ}$; by 6 P.M. it was under $102^{\circ}$; and by 7 P.M. it had reached its lowest point $-100 \cdot 5^{\circ}$. On the morning of the following day the temperature was normal. Quantities necessary to produce the effect have varied from 3 c.c. to 7 c.c. With two of the patients, although undoubtedly tuberculous, I failed to obtain positive results. A control experiments I injected normal sweat into tuberculous animals and phthisical sweat into non-tuberculous. If the quantity of sweat did not exceed 10 c.c. no detectable effect on the temperature resulted except the initial fall which invariably occurs in small rodents as the result of the shock of the injection. With 5 c.c. or 6 c.c. the temperature was always sub-normal for several hours. There seems to be no reason for doubt, therefore, that the night sweat of phthisical patients contains tuberculin in notable quantity. To my mind this proves that these sweats are eliminative efforts on the part of the tissues, and that therefore the very worst treatment which we can adopt for our patients is that of attempting to arrest the sweating by atropine, picrotoxin, zinc oxide, or other antisudorific. The rational line of treatment seems rather to be the encouragement of the sweating by warm blankets, hot bottles, \&c., and the enveloping of the patient in some garment of highly absorbent roaterial, such as a nightshirt of Jaeger's texture or other woollen vestment of like nature.

Effects of normal sneat upon animals.-Before proceedir $\mathrm{g}$ to detail the results of the injection of sweat containing other bacterial toxins it may be asked what are the physiological effects of normal sweat? Subcutaneously enormous quantities from a bealthy man (from 60 c.c. to 100 c.c. or more) may be injected without producing any obvious effect upon rodents. Queirolo, for instance, in fourteen experiments was unable to excite any symptoms whatever, however great the doses employed. As a rule not even local irritation or subsequent suppuration is observed, though the liquid is very far from being aseptic. With very large but submortal doses (from 40 c.c. to 50 c.c.) given intravenously the animals may sometimes present mild rise of temperature prostration, or slight paresis of the hind legs. Arterial pressure may be markedly lowered and loss of weight follows in the next three or four days. It may be noted, however, that no pathological effects of any sort are elicited by the introduction of such small quantities as those mentioned above as used in the examination of phthisical sweat. With regard to the intravenous injection of large quantities my results agree entirely with those of Marrojanni, Capitan and Gley, Queirolo and Cabitto, and are in opposition to Arloing's. I have found that at least from $60 \mathrm{c} \mathrm{c}$. to $100 \mathrm{c.c}$. per kilogramme of animal are necessary to produce death in rabbits. This occurs at the end of from thirty-six to seventy-two bours, but such an event is distinctly rare. Subcutaneously it is impossible to kill animals with normal sweat, however enormous the dose. No rise of temperature with subcutaneous injection of normal sweat occurs unless quantities of at least from 30 c.c. to 40 c.c. are introduced. Capitan and Gley ${ }^{4}$ in one experiment they quote noticed no pyrexia even after 45 c.c. intravenously followed by $50 \mathrm{c}$ c. subcutaneously in the same animal. Dogs are more sensitive to the toxic action of sweat than any other laboratory animal.

Pneunonic sneat.-The results are quite otherwise with pathological sweats. I bave employed the sweat from four patients with acute lobar pneumonia collected from the beginning of the crisis onwards during the next few hours. The rabbit and the moure, the animals most susceptible to infection with the pneumococcus, seemed to be the most suitable subjects for testing this variety of sweat upon. From $\frac{1}{2}$ c.c. to 1 c.c. of pneumonic sweat injected into mice made the animals very ill. Their respiration became accelerated. They sat " hunched up" in a corner of their cage looking precisely like creatures which bave been inoculated with living cultures of the pneumococcus itself. The sweat of one patient, a man in Stephen Ward, Guy's Hospital, killed two mice with the above symptoms in doses of $\frac{1}{2}$ c.c. and 1 c.c. respectively. The critical sweats of pneumonia have, in fact, yielded positive results in every case I have examined and the animals inoculated therewith recall the exact clinical picture of pneumococcus septicæmia. Normal sweat in the same doses has no effect upon mice. Six c.c. of pneumonic sweat also profoundly affected a rabbit and sent up its temperature from $102.8^{\circ}$ to $104.6^{\circ}$, this rise being asscciated with marked acceleration of respiration.

Diphtheria. - I have only twice been fortunate enough to obtain sweat from patients suffering from diphtheria. One of these was a child with laryngeal obstruction and urgent dysprcea. Sweat was standing in beads upon her forehead and I obtained a large quantity in a few minutes. 4 c.c. and 6 c.c. respectively of this were injected in to two small guineapigs of 220 and 260 grammes weight. In twelve hours each had a marked local swelling at the site of inoculation and they had lost 12 and 15 grammes respectively in weight. One was killed at the end of forty-eight hours and showed on section a large infiltration of a gelatino - serous character identical with that observed in experimental diphtheritic intoxication. The suprarenals also seemed somewhat redder than normal, though I cannot speak positively upon this point. The other guinea-pig continned losing weight for ten days and then recovered with necrosis of skin over its abdomen, exactly as occurs after introduction of the pure toxin. Skin necrosis never follows injection of healthy sweat. This observation as to the elimination of diphtheritic toxin through the skin I have confirmed by an experiment in another direction. A horse which was undergoing immunisation at the British Institute of Preventive Medicine with the object of obtaining an antitoxic serum exhibited at first a very unusual result of the treatment. On its arrival it was \&ubjected to a preliminary testing with mallein and in an hour's time became covered 
with a profuse urticarial eruption. The same thing happened within half an hour of its first inoculation with pure diphtheria toxin. After its second dose of toxin in addition to the urticaria it was thrown into a profuse perspiration. I thereupon shaved an area of skin upon its flanks of about eight inches in diameter and collected some of the moisture in one of the bulbed tubes before described. This was injected into a guinea-pig and gave rise to a marked and typical local reaction.

Tetanus.-I have obtained sweat from one patient with acute traumatic tetanus, but though a considerable quantity was injected I obtained no positive effects.

Conclusions. - The above experimental evidence seems to me to have a practical bearing upon therapeutics inasmuch as it furnishes a rational basis for the old empirical method of treatment-viz., that of "sweating a ferer." The artificial encouragement of the sweating no doubt assists in the elimination of the toxin by way of the skin leaving less oehind to poison the tissues. I hope in a further communication to detail the results of the experimental examination of the sweat of patients with acute rheumatism, which $I$ hope Will assist in throwing some light upon the nature of the cineumatic toxin.

Sudbury, Middleser.

ON THE SERUM DIAGNOSIS OF TYPHOID FEVER, WITH ESPECIAL REFERENCE TO THE BACILLUS OF GÄRTNER AND ITS ALLIES

\author{
BY HERBERT E. DURHAM, M.A., M.B. CAMB., \\ F.R C.S. ENG.,
}

GROCHRS' RESEARCH SCHOLAR.

(From the Pathological Laboratory of the University of Camöridge.)

ELSEWHERE I have called attention to the possible mportance in the seram diagnosis of typhoid fever of Landsteiner's observation made in Professor Gruber's Institute that Gärtner's bacillus reacts positively with typhoid serum. That the same occurred in the case of the serum of typhoid fever patients I demonstrated at Liverpool in February, 1897.' It seemed that further investigation should be made upon this point, and the recent epidemics at Maidstone, clifton, and King's Lynn have afforded an opportunity for inquiry. I must here express my sincere thanks to Mr. P. Adams and Dr. Poole, of Maidstone, Dr. Lansdown, of Clifton, and Dr. Plowright and Dr. Sumpter, of Lynn, for most kindly giving me facilities for obtaining serum and also for sending me many samples at their own personal inconvenience.

Before entering into the question of the serum reactions it will be well to make a few preliminary remarks upon the bacillus enteritiỏis (Gärtner), an organism which has hardly received the attention that it deserves in this country and which must not be confounded with the unhappily named bacillus enteritidis sporogenes of Dr. Klein. Authors have been in the habit of grouping the typhoid and colon bacilli in rather a promiscuous way-the "typhoid-like" of one observer being a "colon-like" to another.

The whole class may be divided into three groups:-(1) the Eberth group includes the typhoid bacillus and its near allies (these are almost unknown); (2) the Gärtner group includes bacillus enteritidis and its near allies (these have not yet been sufficiently worked out); and (3) the Escherich group includes the true bacillus coli and its near allies (these also have not yet been sufficiently worked out). I do not propose to enter here into the discussion of further groups of bacilli which in some respects resemble those of Gromp 3, though widely differing from those of Groups 1 and 8 .

In the literature of the subject it appears probable that members of the Gärtner group have been confounded with those of Croup: 1 and 2 . However, they are not very difficalt to distioguish in practice. In Germany, Holland, and Belgium, a number of outbreaks of "meat poisoning" have been carefully investigated; in many of these the causation of illness has been ascribed to certain bacilli or their toxic products, which bears a striking family resem. blance to the original account of Giurtner; the individual differences which have been noticed are apparently comparatively of little importance. These bacilli have been isolated from the infected meat (often in the form of "German sausage"), vomit, and frees, as also from the organs in fatal cases. A perusal of Dr. Güntber's paper will show what care and patience may be required in working out a case of "meat poisoning" with success.

In the accompanying table in which the three groups are contrasted the description refers to characters which are presented by the actual bacillus of Gärtner at my disposal; so far as Gärtner's description goes, it is at variance only in the number of flagella (from four to eight) and the production of gas bubbles in lactose media. At the time when he wrote the technique of cilia staining was not so perfect as at present. $^{2}$ Other writers as well as Gärtner have given their bacilli credit of producing gas, though small in amount, in lactose media. As they did not use media free of muscle sugar ("bouillon") their statements are without value. The terms "paratyphoid" and "paracoli," about the relative merits of which there has been some discussion between Widal and Achard, do not appear to me to be at all appro. priate; the group is evidently quite distinct from both typhoid and coli. Moreover, there are "Escherich-like" bacilli which are also unable to "ferment" lactose.

It will be observed that the bacillus Gärtner only differs from the bacilius Eberth in being able to produce gas bubbles in the presence of glucose (and muscle sugar), in possessing a greater power of overcoming the pre liminary acid formed in the presence of glucose or lactose, and in reducing power. The expression "producing gas bubbles" is nsed bere advisedly, since Hesse (vide Lösener) has shown that the typhoid bacillus can produce much gas $\left(\mathrm{CO}_{2}\right)$ though not as visible bubbles. $\left(\mathrm{H}_{2} \mathrm{~S}\right.$ is also evolved.) The eventual alkali formation is dependent upon the amount and kind of sugar, the presence of oxygen, and the initial alkalinity of the medium. If twenty-four hours old cultures of neutral litmas-whey are exactly neutralised it will be fonnd that the typhoid and Gärtner bacilli never become acid again, whilst the bacillus coli will form as much acid as it had aiready done before; although there is still plenty of sugre the two former do not decompose it into acid. Exactly the reverse is the case when cultures in 2.0 per cent. peptone, 0.1 per cent. glucose are neutralised. The great power of alkali production possessed by the bacillus Gärtner is no doubt causally related to the fact that it has a tendency to surface growth. Cobbett has shown that surface growth is necessary for alkali production by the diphtheria bacillus. To the Gärtner group belong the bacilli described by van Ermenghem, Fischer. Gaffky and Paak, Basenau, Cotta, Kaensche, Karlinsky, Günther, \&c. Lubarsch bas described a bacillus from a newly born child (without data concerning flagella, gas, or indol) which Gärtner reported on as being identical with his bacillus; however, it differs in that it clots milk (vide Gärtner's original description). Petruschky's bacillus fæcalis alkaligenes possibly belongs here also, so far I have been nnable to obtain a culture to examine, the original description not being sufficiently detailed. The "typhoid" bacilli of certain authors "which have the power of gas production" almost certainly belong here too; also not improbably the cases of "septicæmic typhoid fever" described in France, in which "death occurred before typical typhoid lesions bad been developed" were in reality due to a bacillus of this group and not to what we recognise as Fberth's bacillas. The different results given in the matter of reducing power ars also cleared up to some extent by non-appreciation of this group by the several writers. My observations are quite in harmony with those of Germano and Maurea, in that I have yet to see a true typhoid bacillus which reduces sulphindigotate of soda as rapidly as the bacillus coli does. Another source of difference in the results lies in the constitution of the media, more especially in the presence of sugars, and natnrally, also, the contact with oxygen. This I hope to deal with more thoroughly elsewhere.

There is yet one more question which is cleared up on the

2 I am now quite convinced that this diserepancy is due to faulty staining method, since the culture just received trom Dr. Günther, which is described to possess from two ta five flagella when stained by Löffler's method, shows from ten to fourteen or more in specimens prepared by that of van Ermenghem. (Specimens were made from fresh four-hour and twenty-hour cultures directly after the receipt of the culture.) 